\title{
TRANSFORMAÇÃO MARTENSÍTICA INDUZIDA POR DEFORMAČÃO PLÁSTICA DURANTE LAMINAÇÃO CRIOGÊNICA EM AÇO INOXIDÁVEL 304L *
}

\author{
Juciane Maria Alves ${ }^{1}$ \\ Luiz Paulo Mendonça Brandão ${ }^{2}$ \\ Andersan dos Santos Paula ${ }^{3}$
}

\begin{abstract}
Resumo
O aço inoxidável austenítico metaestável 304L, aço TRIP, é suscetível à transformação de fase austenita-martensita $\left(\gamma \rightarrow \alpha^{\prime}\right)$ induzida por plasticidade, a intensidade desta transformação é influenciada pela temperatura. Devido a isto, o objetivo deste trabalho foi induzir a transformação $\left(\gamma \rightarrow \alpha^{\prime}\right)$ através da laminação à temperatura criogênica, mais próxima da temperatura Mi do material. Para avaliação da transformação de fase utilizou-se a técnica de difração de raios-X e os resultados mostraram a obtenção de amostras 100\% martensíticas. Devido à suscetibilidade deste material sofrer transformação $\left(\gamma \rightarrow \alpha^{\prime}\right)$ por deformação plástica avaliou-se a influência da preparação metalográfica (via polimento mecânico, químico e eletrolítico) sobre às análises quantitativas. O polimento eletrolítico se mostrou a forma mais promissora às análises quantitativas, pois permitiu a quantificação de pequena fração volumétrica de austenita retida..
\end{abstract}

Palavras-chave: Temperatura Mi; Preparação Metalográfica; DRX.

\section{STRAIN-INDUCED MARTENSITIC TRANSFORMATION DURING CRYOGENIC ROLLING IN 304L STAINLESS STEEL}

\begin{abstract}
The 304L austenitic stainless steel, TRIP steel, is susceptible to the austenitemartensite $\left(\gamma \rightarrow \alpha^{\prime}\right)$ phase transformation induced by plasticity, the intensity of this transformation undergoes is influenced by temperature. Due to this, the objective of this work was to induce the transformation $\left(\gamma \rightarrow \alpha^{\prime}\right)$ through the cryogenic rolling, close to the Mi temperature of the material. The X-ray diffraction technique was used to evaluate the phases transformation and the results showed $100 \%$ martensitic samples. Due to the susceptibility of this material undergo $\left(\gamma \rightarrow \alpha^{\prime}\right)$ transformation by plastic deformation, the influence of the metallographic preparation (through mechanical, chemical and electrolytic polishing) was evaluated on the quantitative analyzes. The electrolytic polishing was more promising to the quantitative analysis, since it allowed the quantification of a small volumetric fraction of retained austenite.
\end{abstract}

Keywords: $M_{s}$ Temperature; Metallographic Preparation; XRD.

1 Química, M.Sc, Doutoranda em Ciência dos Materiais no Instituto Militar de Engenharia - IME, Rio de Janeiro, RJ, Brasil.

2 Engenheira Metalúrgica, D.Sc., Professora Adjunta, Seção de Engenharia Mecânica e de Materiais, Instituto Militar de Engenharia - IME, Rio de Janeiro, RJ, Brasil; e orientadora credenciada da PPGEM/UFF, Volta Redonda, RJ, Brasil.

3 Engenheiro Metalúrgico, D.Sc., Professor Associado, Seção de Engenharia Mecânica e de Materiais, Instituto Militar de Engenharia - IME, Rio de Janeiro, RJ, Brasil. 


\section{INTRODUÇÃO}

Aços inoxidáveis austeníticos metaestáveis 304L são suscetíveis à transformação de fase induzida por plasticidade devido ao Efeito TRIP. O mesmo quando submetido à deformação por ensaio de tração, laminação entre outros processos/modos de deformação pode sofrer transformação $\left(\gamma \rightarrow \alpha^{\prime}\right)$. Este aço faz parte de uma geração de aços inoxidáveis austeníticos de elevada resistência [1]. A transformação de fase da austenita metaestável $(\gamma)$ em martensita $\left(\alpha^{\prime}\right)$ depende de condições importantes como composição química da liga, temperatura do ensaio, taxa e nível de deformação plástica, além do modo de deformação. A composição química da liga afeta fatores importantes, intrínsecos, à transformação de fase como a temperatura de início de formação de martensita $\left(\mathrm{M}_{\mathrm{i}}\right)$ (Equação 1) e a temperatura $\mathrm{Md}_{30}$, na qual $30 \%$ da deformação imposta por tensão induz à transformação de $50 \%$ de austenita em martensita [2, 3, 4].

$$
M_{i}\left({ }^{\circ} \mathrm{C}\right)=1305-1665(\% \mathrm{C}+\% \mathrm{~N})-28(\% \mathrm{Si})-33(\% \mathrm{Mn})-42(\% \mathrm{Cr})-61(\% \mathrm{Ni})
$$

A transformação $\left(\gamma \rightarrow \alpha^{\prime}\right)$ em aço TRIP é fortemente influenciada pela temperatura, pois a deformação plástica, de caráter irreversível, provoca elevação da temperatura interna do material e dependendo da taxa com a qual o material seja deformado poderá não haver tempo hábil para a dissipação do calor para o meio, processo denominado adiabático. A temperatura torna-se maior, quanto maior a quantidade de deformação ou taxa de deformação as quais o material seja submetido. Dependendo da intensidade destes efeitos a transformação de fase poderá ser suprimida $[5,1,6]$. De fato, a deformação plástica inicia-se com o deslizamento planar de discordâncias, porém com aumento da deformação ou taxa de deformação promove-se o deslizamento não planar, com acúmulo de discordâncias. Este fato resulta no aumento de calor com aquecimento local e, desta forma, a transformação é inibida pela redução de sítios de nucleação [1]. Baixas temperaturas favorecem a formação de martensita- $\alpha$ ' diminuindo desta forma a fração volumétrica de austenita [7]. Devido a influência de cada um dos fatores citados anteriormente, os mecanismos da transformação martensítica como tensão assistida e transformação induzida por deformação podem ser diferentes para cada material que sofre transformação austenita-martensita.

Este trabalho teve como objetivo induzir a transformação martensítica em um aço inoxidável austenítico 304L através da laminação simétrica em uma temperatura criogênica, próxima à temperatura de início de formação de martensita (Mi). Em paralelo, foram avaliadas diferentes técnicas de preparação metalográfica como polimento mecânico, químico e eletrolítico com o objetivo de avaliar possíveis influências sobre a quantificação de fases. Utilizou-se a técnica macroscópica Difração de Raios-X para realizar o estudo quantitativo.

\section{MATERIAL E MÉTODOS}

O material em estudo consistiu em chapa de aço inoxidável austenítico 304L, laminado a quente, com espessura de $6,37 \mathrm{~mm}$. Sua composição química apresentada na Tabela 1. Este material foi doado por Aperam South América. 
Tabela 1. Composição química aço inoxidável 304L em estudo.

\begin{tabular}{ccccccccc}
\hline \multicolumn{7}{c}{ Elemento (\% em peso) } \\
\hline $\mathrm{C}$ & $\mathrm{Mn}$ & $\mathrm{Si}$ & $\mathrm{P}$ & $\mathrm{S}$ & $\mathrm{Cr}$ & $\mathrm{Ni}$ & $\mathrm{Mo}$ & $\mathrm{Al}$ \\
0,0192 & 1,2686 & 0,3770 & 0,0352 & 0,0026 & 18,0722 & 8,0082 & 0,0958 & 0,0032 \\
$\mathrm{Cu}$ & $\mathrm{Co}$ & $\mathrm{V}$ & $\mathrm{Nb}$ & $\mathrm{Pb}$ & $\mathrm{B}$ & $\mathrm{Ti}$ & $\mathrm{Sn}$ & $\mathrm{N}$ \\
0,1557 & 0,1712 & 0,0802 & 0,0056 & 0,0010 & 0,0006 & 0,0060 & 0,0062 & 0,0541 \\
\hline
\end{tabular}

O aço 304L foi submetido à laminação simétrica à temperatura criogênica (LTC) próxima a $-118^{\circ} \mathrm{C}$. Esta temperatura foi estimada através da Equação (1), temperatura de início de formação de martensita $\left(\mathrm{M}_{\mathrm{i}}\right)$, que depende da composição química do material. A partir de testes preliminares, considerando-se o tempo de retirada da amostra do nitrogênio líquido, posicionamento da mesma sobre a mesa do laminador e realização da laminação, determinou-se que esta seria resfriada a uma temperatura de $-125^{\circ} \mathrm{C}$. Logo, a amostra ao passar entre os cilindros de laminação, a cada passe, estaria aproximadamente a $-118^{\circ} \mathrm{C}$. Foram realizados ao todo 25 passes de laminação com reduções de aproximadamente $6 \%$ em cada passe, resultando em amostras com 20, 40, 60 e $80 \%$ de redução.

As amostras foram submetidas a diferentes métodos de preparação metalográfica como polimento mecânico, químico e eletrolítico. Na etapa de preparação via polimento mecânico as amostras foram submetidas ao lixamento com lixas de 600, 800 e 1200 mesh e polidas em pasta de diamante com granulometrias de 3,0; 1,0 e $0,25 \mu \mathrm{m}$, utilizando-se panos de polimento compatíveis às granulometrias das pastas. O lixamento foi realizado em lixadeira automática, AROTEC - ARAPOL $2 \mathrm{~V}$, com baixa velocidade e o polimento realizado manualmente a fim de minimizar os efeitos da tensão superficial no material. $O$ polimento químico foi realizado com uma solução composta por $0,7 \mathrm{ml}$ de ácido fluorídrico (concentração $40 \%$ P.A), 0,3 $\mathrm{ml}$ de ácido nítrico (concentração 65\% P.A) em 3,0 $\mathrm{ml}$ de água destilada. A temperatura da solução ficou entre 50 e $60^{\circ} \mathrm{C}$, com uso de uma placa de aquecimento QUIMIS Q261-12. O tempo de imersão das amostras foi entre 1 minuto e 10 segundos, onde a amostra como-recebida (CR) necessitou de um maior tempo de reatividade na solução, já o tempo de imersão das demais amostras foi menor de acordo com o incremento do percentual de martensita. Já o polimento eletrolítico foi realizado com solução de $20 \%$ de ácido perclórico (concentração $70 \%$ P.A) em álcool etílico absoluto. A fonte utilizada (POLITERM - DC POWER SUPPLY HY3003E-3) operou com voltagem de $20 \mathrm{~V}$ e o tempo de imersão das amostras ficou entre 7 e $10 \mathrm{~s}$. Em função do incremento de martensita nas amostras o tempo de imersão foi menor resultando em uma superfície ótima de polimento.

Para avaliação de fases submeteu-se as amostras à difração de raios- $X$ utilizandose o difratômetro modelo X'PERT PRO MPD da PANalytical, com o software Data Colector - Absolute Scan. Foi adotada a configuração foco linha para a obtenção de difratogramas com boas intensidades, voltagem e corrente do equipamento de $40 \mathrm{kV}$ e $45 \mathrm{~mA}$, respectivamente, e utilizou-se filtro de ferro para o tubo de cobalto. Os parâmetros de medida para cada amostra foram ângulos iniciais e finais de $45 \mathrm{e}$ $130^{\circ}$, tamanho do passo de $0,030^{\circ}$, tempo por passo de $200 \mathrm{~s}$, o que levou a um tempo total de análise de 38 minutos e 33 segundos. O software de análise quantitativa a partir do método de Rietveld foi o TOPAS versão 4.2. 


\section{RESULTADOS E DISCUSSÃO}

A evolução da transformação de fases $\left(\gamma \rightarrow \alpha^{\prime}\right)$ após laminação criogênica, com reduções de 20, 40, 60 e $80 \%$, assim como o ajuste entre os picos teóricos e experimentais através do método de Rietveld são apresentados na Figura 1. Os resultados quantitativos desta transformação de fase, após polimento eletrolítico, são apresentados na Figura 2.

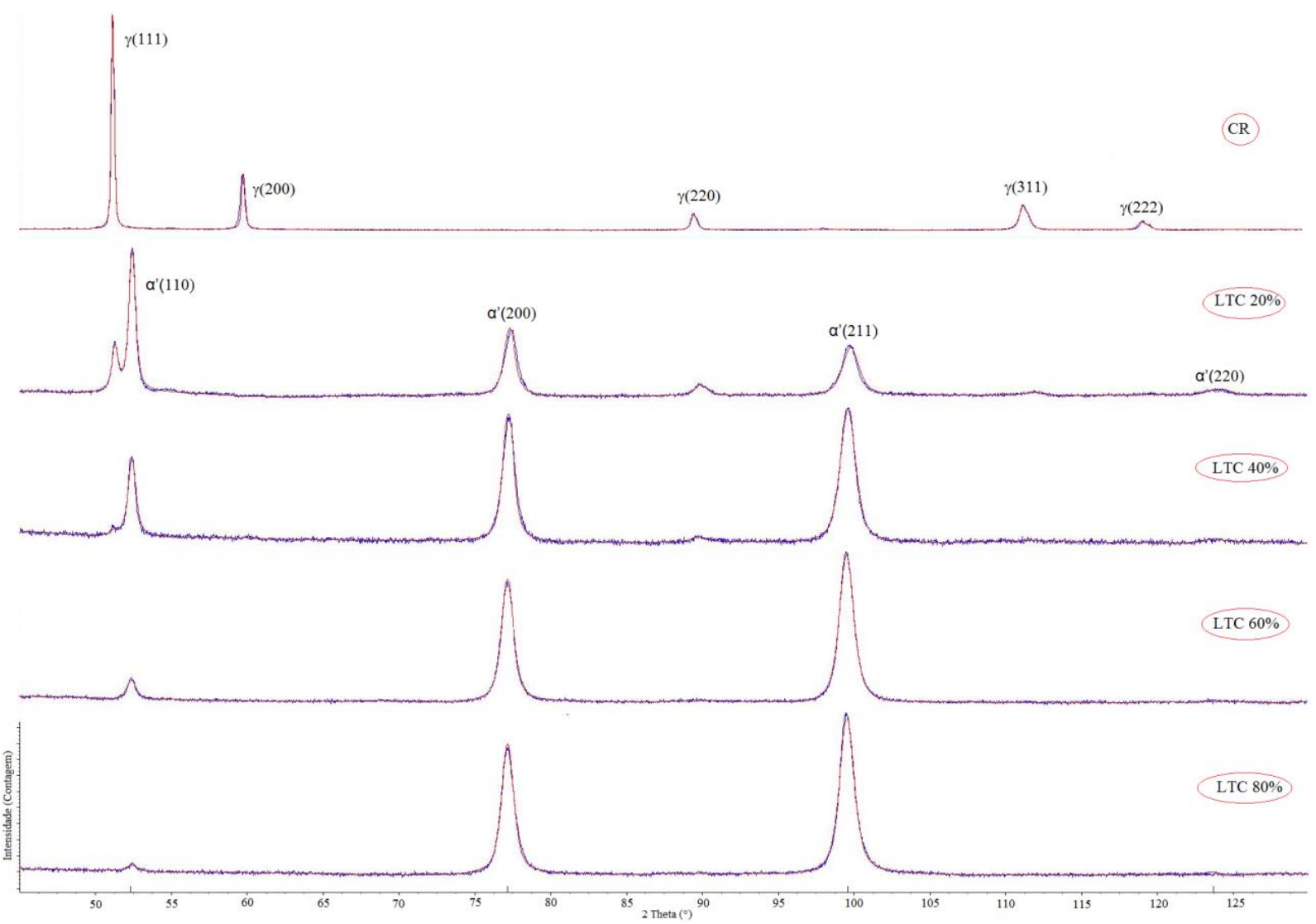

Figura 1. Difratogramas associados a evolução da transformação de fase após laminação à temperatura criogênica com reduções de $20,40,60$ e $80 \%$ após polimento eletrolítico.

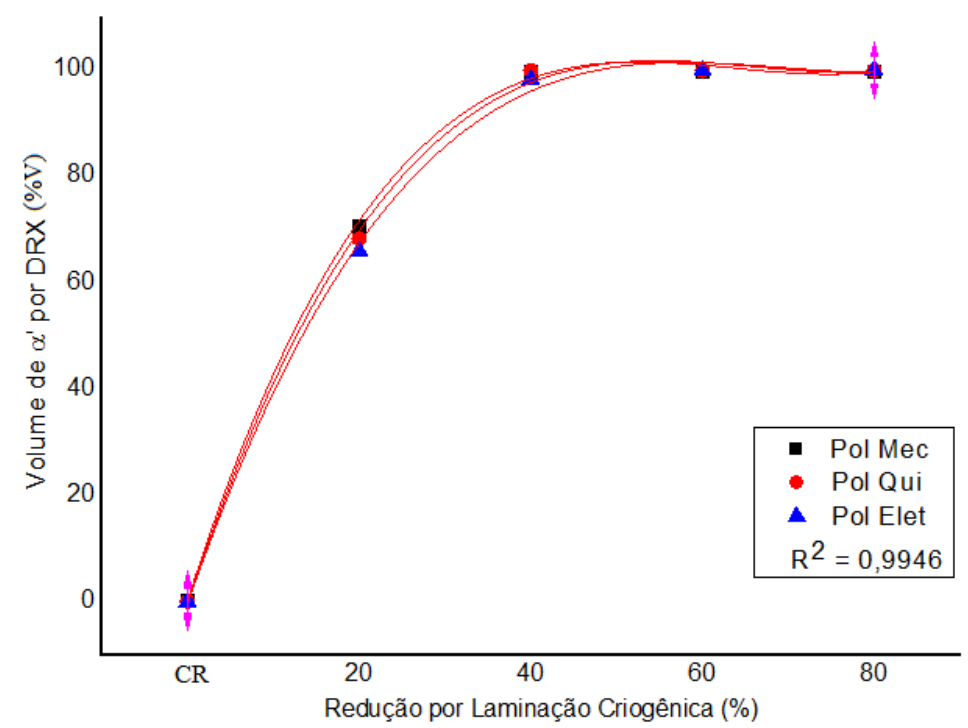

Figura 2. Quantificação de $\left(\gamma \rightarrow \alpha^{\prime}\right)$ a partir de medidas por DRX após polimento eletrolítico. 
São apresentados na Figura 3 os resultados das medidas de DRX obtidos a partir da preparação metalográfica via polimento mecânico, químico e eletrolítico das amostras CR e com reduções de 20, 40, 60 e 80\% em laminação criogênica.

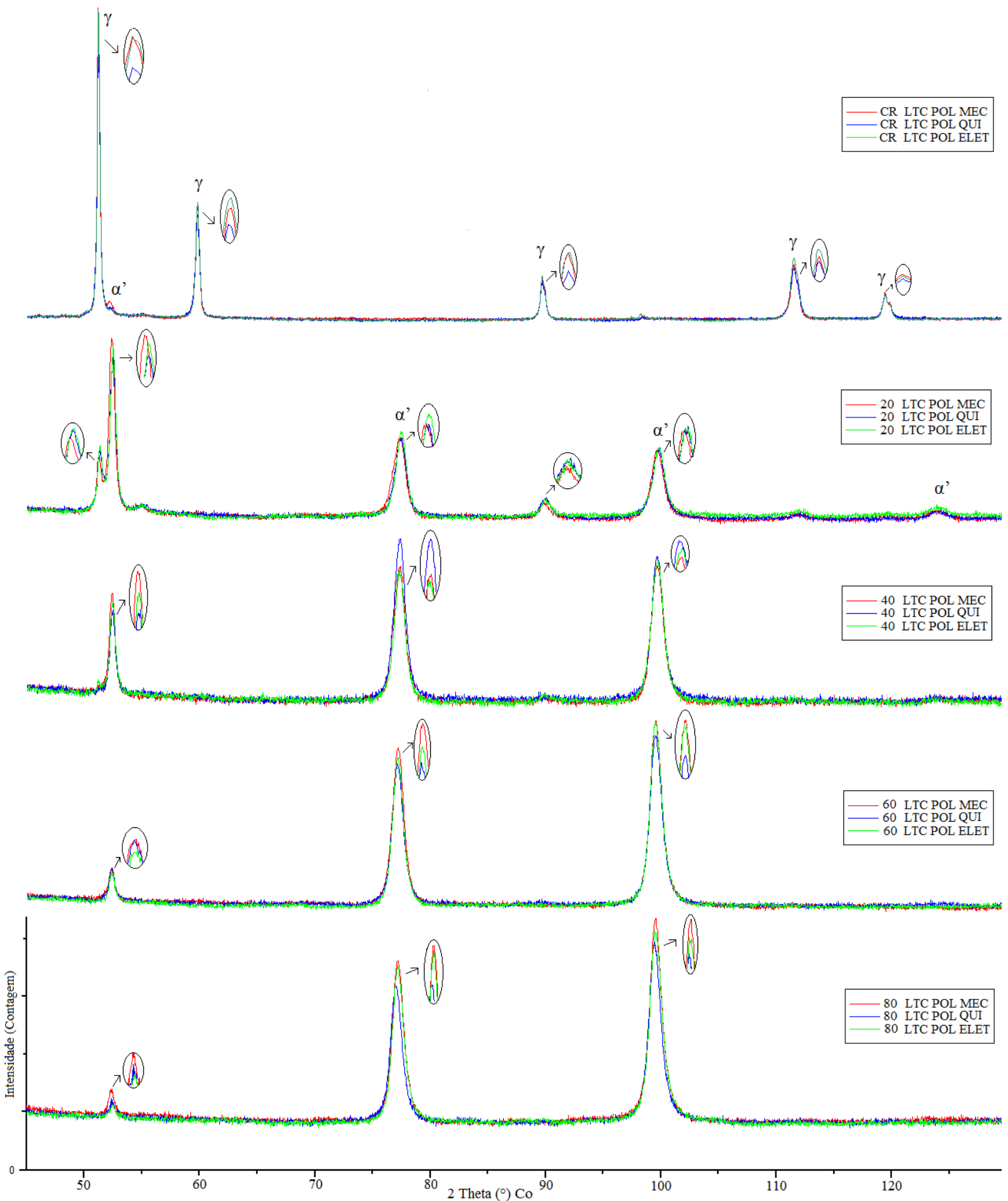

Figura 3. Preparação metalográfica via polimento mecânico, químico e eletrolítico da amostra CR e de amostras com 20, 40, 60 e 80\% de redução após laminação criogênica.

A evolução da transformação martensítica apresentada na Figura 1 pode ser claramente observada através da redução de intensidade dos picos de austenita $\gamma((111),(200),(220),(311),(222))$ e pelo incremento da intensidade dos picos de 
martensita $\alpha^{\prime}((110), \quad(200), \quad(211), \quad(222), \quad(220))$ em seus respectivos planos cristalográficos de difração, após reduções por laminação nos percentuais de 20, 40 60 e $80 \%$. Inicialmente com a redução de $20 \%$ observou-se intensos picos de martensita referentes aos planos cristalográficos difratados $\alpha^{\prime}((110),(200)$ e (211)). Após preparação metalográfica via polimento mecânico, químico e eletrolítico foram observados percentuais volumétricos de martensita de 70,73; 68,35 e 66,02\%, respectivamente. Com a redução de $40 \%$ houve um incremento dos picos de martensita referentes aos planos difratados $\alpha^{\prime}((200)$ e (211)) restando um pequeno pico de austenita correspondendo ao plano cristalográfico $\gamma(111)$. Quantitativamente observou-se a partir do polimento mecânico e químico 100\% de a', e 98,28\% desta fase foi observado após o polimento eletrolítico. Após a redução de $60 \%$ os difratogramas apresentaram picos apenas de martensita, porém após a preparação metalográfica via polimento eletrolítico foi possível quantificar $1,72 \%$ de austenita retida em volume. Já os resultados obtidos a partir de polimento mecânico e químico mostraram $100 \%$ de martensita nesta redução por laminação. Na redução de $80 \%$ os difratogramas apresentaram incremento na intensidade dos picos de a' referentes aos planos $\alpha^{\prime}\left((200)\right.$ e (211)) e uma redução no pico $\alpha^{\prime}(110)$. Ressalta-se que o valor estatístico referente à qualidade do ajuste (GOF) entre os picos teóricos e experimentais, linha azul e vermelha, ficou entre 1,83 e 1,09. Este valor está dentro do aceitável, uma vez que o mesmo deve ser igual ou menor que 2 considerando-se um bom refinamento com o software TOPAS.

Através da Figura 2 pode ser observado o incremento do volume de martensita em função da deformação plástica em baixa temperatura. De acordo com os resultados, a amostra CR apresentou uma fração residual de $1,65 \%$ de martensita após polimento eletrolítico. Esta fração martensítica superficial possivelmente é resultante de resíduo da preparação metalográfica, na etapa de lixamento devido às tensões cisalhantes, ou resultante de alguma operação de planificação da amostra após seu desbobinamento (por parte do fornecedor do material para o estudo).

Em função dos diferentes tipos de preparação metalográfica como polimento mecânico, químico e eletrolítico observou-se, de acordo com a Figura 3, que houve diferença nas intensidades dos picos de difração de cada fase, austenita e martensita, associadas aos planos cristalográficos $\mathrm{Y}((111),(200),(220),(311),(222))$ e a'((110), (200), (211), (222), (220)). Como pode ser observado, o comportamento das intensidades dos picos não segue uma determinada tendência em função do tipo de polimento. Mas através da análise quantitativa por difração de raios- $X$ foram obtidos diferentes percentuais volumétricos das fases austenita e martensita, após realização do polimento mecânico, químico e eletrolítico. As análises quantitativas mostraram que o percentual volumétrico de martensita foi maior após a preparação por polimento mecânico e menor após polimento eletrolítico, e um valor intermediário com o polimento químico. A diferença quantitativa em função dos distintos métodos de preparação metalográfica deve-se à eficácia de cada método em remover martensita superficial mencionada anteriormente. Desta forma, o polimento eletrolítico apresenta-se como aquele mais promissor para o estudo quantitativo da transformação de fases $\left(\gamma \rightarrow \alpha^{\prime}\right)$, pois possui maior capacidade de reduzir/remover a camada de martensita superficial.

Análises de transformação de fase $\left(\gamma \rightarrow \alpha^{\prime}\right)$ por DRX foram realizadas com a amostra como-recebida para verificação de possível transformação induzida apenas por temperatura. Os resultados da amostra imersa em nitrogênio líquido por 5 minutos e 1 hora mostraram que não houve transformação martensítica induzida por temperatura. Logo, a transformação $\left(\gamma \rightarrow \alpha^{\prime}\right)$ ocorreu em resposta à deformação 
plástica em laminação, favorecida pela temperatura criogênica, mais próxima a temperatura Mi do aço 304L.

\section{CONCLUSÃO}

A temperatura criogênica, próxima à temperatura $(\mathrm{Mi})$, utilizada no processo de laminação simétrica, favoreceu a obtenção de alto percentual de a' já a partir de $20 \%$ de redução, e com reduções de 60 e $80 \%$ obteve-se $100 \%$ de martensita. Utilizando-se diferentes métodos de preparação metalográfica como polimento mecânico, químico e eletrolítico foi possível observar claramente a influência dos mesmos sobre a quantificação de fases em um aço TRIP 304L. O polimento eletrolítico se mostrou de forma mais promissora à quantificação de fases, pois através do mesmo foi possível quantificar pequena fração volumétrica de austenita retida. Observou-se que o tempo de imersão das amostras em polimento químico e eletrolítico torna-se menor conforme o percentual de martensita aumenta. Como verificado a partir de diferentes tempos de resfriamento da amostra CR em nitrogênio, esta transformação de fase ocorreu em resposta à deformação plástica em laminação, favorecida pela temperatura. Pois a força motriz, associada a tensão aplicada foi suficiente para a obtenção de $100 \%$ de $\alpha^{\prime}$ a aproximadamente $-118^{\circ} \mathrm{C}$. Desta forma, a partir de observações na literatura, conclui-se que este trabalho apresenta excelentes resultados da transformação de fases $\left(\gamma \rightarrow \alpha^{\prime}\right)$ via laminação criogênica avaliando-se também diferentes métodos de preparação metalográfica.

\section{AGRADECIMENTOS}

Os autores agradecem à Aperam South América pelo fornecimento do material para estudo e à FAPERJ pela bolsa Aluno Nota 10. A.S. Paula agradece ao CNPq a bolsa de produtividade a pesquisa PQ-2 (Processo: 307798/2015-1).

\section{REFERÊNCIAS}

1 Shen, YS, Li XX, Sun X, Wang, YW, Zuo L. Twinning and martensite in a 304 austenitic stainless steel. Materials Science and Engineering A. 2012;552:514522.

2 Cina BJ. Iron Steel Inst. (1954):791.

3 ANGEL, T.; Formation of Martensite in Austenitic Steels, J. Iron Steel Inst., 5(1954), pp.165-174.

4 Hedayati A, Najafizadeh A, Kermanpur A, Forouzan F. The effect of cold rolling regime on microstructure and mechanical properties of AISI 304L stainless steel. Journal of Materials Processing Technology. 2010;210:1017-1022.

5 Yoo SW, Lee CS, Park WS, Kim MH, Lee JM. Temperature and strain rate dependent constitutive model of TRIP steels for low-temperature applications. Computational Materials Science. 20;50:2014-2027.

6 Pruger S, Seupel A, Kuna M. A thermomechanically coupled material model for TRIP-steel. International Journal of Plasticity 2014;55:182-197.

7 Nagy E, Mertinger V, Tranta F, Sólyom J. Deformation induced martensitic transformation in stainless steels. Materials Science and Engineering $A$. 2004(378):308-313. 